\title{
Use of Dacron prosthesis in esophageal diverticula in a dog - a case report
}

\author{
Aurel Nicolae Muste ${ }^{1}$, Andrei Tănase², Marius Mihai Muste¹, Florin Ioan Beteg \\ ${ }^{1}$ University of Agricultural Sciences and Veterinary Medicine, Faculty of Veterinary Medicine, \\ Department of Surgery, Cluj-Napoca, Romania \\ ${ }^{2}$ University of Agricultural Sciences and Veterinary Medicine Bucharest, Faculty of Veterinary Medicine, \\ Department of Surgery, Romania \\ Received September 24, 2013 \\ Accepted May 28, 2014
}

\begin{abstract}
A one-and-a-half-year-old male dog of the Caniche breed was examined for repeated regurgitation episodes. At clinical examination food or liquid regurgitation episodes were reported at different times after food ingestion. The esophagus was visualized with the videoendoscope and then examined on liquid barium sulphate. The assessment revealed the existence of two cervically and mediastinally (intrathoracically) located dilatations. The surgical procedure was based on polyester vascular prostheses also used in human vascular surgery. The surgery was performed in two stages with a 4 week interval in between, without the resection of the dilated walls, with the prostheses positioned and fixed in different ways and with very good post surgery recovery. Eleven months after the surgery the dog's health condition was very good. To our knowledge, this is the first report about these procedures which avoid the resection of a portion of the esophagus wall, thus preventing the occurrence of scars or strictures at this level, and prostheses increase the support and stability of the affected esophagus portion.
\end{abstract}

Canine, esophagus, diverticular dilatation, surgery

One-and-a-half-year-old male dog, Caniche, $6.5 \mathrm{~kg}$, was presented, manifesting repeated food or non food (liquid) regurgitation episodes at different times after food ingestion. A polyester prosthesis was used on the subject. This type of prosthesis features good stability and elasticity, and can work more than 10 years after the implant. It is highly resistant, toxic free, does not cause either intolerance or undesired side effects and reacts well in high flow and high speed areas.

Esophagus diseases in dogs are diagnosed based on regurgitation episodes ocurring mostly after eating. Postprandial regurgitation is common in dogs suffering from esophageal diverticulum (Shaw et al. 1997). Esophageal diverticula are either congenital or inherited as dilatation (sack) of the esophageal wall with lower frequency in dogs. Consequently there are only a few reports on this issue world-wide (Hill et al. 1979). Esophageal diverticula are classified as pulsion and traction diverticula (Shaw et al. 1997) with the remark that pulsion diverticula are incriminated in the epiphrenic area (Pearson et al. 1978).

Authors such as Qin et al. (2003) and Zhang et al. (2008) used polyurethane artificial stent prostheses covered by chitosan-collagen sponge in dogs for esophagus reconstruction purpose.

This report describes the clinical, endoscopic and therapeutic features of a canine patient suffering from double esophageal diverticulum (in the cervical and endothoracic area) in which vascular prosthesis was used for the first time.

\section{Case presentation}

Postprandial regurgitation and vomiting at different times after food ingestion was reported. The portprandial episodes started 3 months before, with a progressive worsening. 
Coughing episodes were reported to precede the regurgitation and vomiting. The owner reported no other significant alteration of the general status.

Clinical examination revealed normal rectal temperature as well as normal heart and respiratory rate. During clinical examination an episode of regurgitation occurred. The haematological and biochemical profile did not change significantly.

Esophagus motility was assessed through esophagoscopy. The esophagus was visualized with the videoendoscope, revealing chronic esophagitis. The esophagus was then examined based on liquid barium sulphate ingestion, $3.5 \mathrm{ml} / \mathrm{kg}$. The assessment revealed the existence of two cervically and mediastinally (intrathoracically) located dilatations. Based on mentioned investigation, differential diagnosis was made with megaesophagus. Radiological and endoscopic features of the diverticula required surgery planning.

Anaesthesia was performed by a combination of diazepam (Diazepam, Terapia Ranbaxy, Cluj Napoca, Romania) at the dose of $0.25 \mathrm{mg} / \mathrm{kg}$ with ketamine chlorhydrate (Ketamidor, Richter Pharma Ag, A4600 Wels, Austria) at the dose of $5 \mathrm{mg} / \mathrm{kg}$, in simultaneous administration, and maintained using isoflurane (Forane, Baxter Healthcare Corporation, Deerfield, IL).

Surgical approach to the cervical area was performed from the dog's left side through a $5 \mathrm{~cm}$ long incision of the skin, connective tissue, and the platysma muscle. Then, the esophagus was isolated from the periesophageal connective tissue by using moist laparotomy sponges.

After exposing the esophagus, the dilated portion was assessed and analyzed, revealing a $6.3 \mathrm{~cm}$ long and $4.2 \mathrm{~cm}$ wide esophagus dilatation (PlateV, Fig. 1).

Careful examination of the esophagus wall revealed a parietal defect consisting of the absence of a muscular layer located in the ventral portion all along the mentioned length.

A $8 \mathrm{~mm}$ thick braided polyester vascular prosthesis with small meshes, also used in human vascular surgery with very good results, was applied all along the affected area.

After preparation and adjustment, the prosthesis was applied onto the esophagus in the dilated portion and fixed using a four-point suture (two points at the ends and two in the remaining distance). Then the prosthesis was wrapped into the esophagus, rolled around the axis (rolling around own axis) until completely covered with the dilated esophagus wall (once and a half, in our case). The esophagus lumen was adjusted to correspond to age and height of patient, i.e. $1.5 \mathrm{~cm}$.

A continuous suture was performed with a non absorbable synthetic thread by passing it all through the esophagus wall so that the vascular prosthesis would remain fixed (hidden) all along the dilated portion and at the same time act as a support on the ventral margin of the esophagus lumen (Plate V, Fig. 2). After building this model of the esophagus, it was placed together with the sutured prosthesis in their natural position, further proceeding to the fixation in a few suture points. The anatomical plans were then reconstructed based on described technique. For three days the dog underwent therapy with parenteral fluid, analgesic (tramadol hydrochlorid - Tramal 50, Grunenthal GmbH, Aachen, Germany), and antibiotics (lincomycin and spectinomycin - Linco-Spectin, Pharmacia NV/SA, Rijkweg 12, B 2870 Puurs, Belgium).

Two weeks after the surgery the subject was clinically and radiologically reexamined. Barium sulphate radiological examination revealed that the prosthetic portion was healed with no diverticula or dilatations present. However, on this occasion we established that the second esophagus dilatation located in the thoracic cavity had increased its volume, requiring a second surgery since the subject's life was in danger. The second surgery was performed 4 weeks after the first one.

General anaesthesia was performed in the same way as for the previous surgery. The operative field was prepared from the dorsal line to the ventral line, and from the shoulder point to the $13^{\text {th }}$ rib. Skin incision was centered on intercostal spaces 4-5, $4 \mathrm{~cm}$ away from 
the chondrocostal joints and over a $5 \mathrm{~cm}$ length. A skin incision was performed, followed by the incision of the subcutaneous connective tissue, thoracic muscles, latissimus dorsi and a part of the scalene muscle. The external oblique muscle was disinserted off the rib and back tractioned so as to have access to the intercostal muscle incision and the parietal pleura. The ribs and soft tissues were protected with moist tampons, and then retracted for access in the thorax. Pulmonary lobes were packed with moist tampons and caudally retracted. The esophagus was identified and the mediastinal pleura was dissected carefully so as not to traumatize the vagus nerve or the surrounding nerves. After the esophagus isolation the prosthesis was prepared. The length of the prosthesis must exceed the parietal defect by $1 \mathrm{~cm}$ both cranially and caudally. For the part of esophagus located intrathoracically, we used a polyester vascular prosthesis of $16 \mathrm{~mm}$ in diameter with the same properties as the one used previously. The prosthesis wall was sectioned all along its length, then it was introduced over the affected esophagus portion so that the prosthesis became a kind of protective "sleeve".

By this procedure the dilated portion was not resected. The prosthesis wall was sutured using a few points so that the margins were in contact and matched. The mediastinal pleura dissected off the esophagus at the beginning of its isolation was then passed over the prosthesis and sutured in separate points with absorbable polyglycolic acid (Dexon II, Sherwood Davis \&Geck). The best anchorage was achieved all along the prosthesis length; the anatomical plans were reconstructed in a reverse order, through suturing of the pleura, intercostal muscles, and thoracic muscles. The sectioned ribs were restored to their physiological position and fixed through osteorrhaphy. Skin suture in separate points and a protective bandage around the thorax were the final steps of the surgery. For 5-7 days after the surgery the dog underwent parenteral fluid therapy along with analgesics and antibiotics.

\section{Discussion}

This disease is classified as rare (Hill et al. 1979) with a post surgery prognosis ranging from favourable to good (Iwasaki et al. 1977). Over time, a large number of methods have been tested, which explains the variety of opinions and techniques relating to the therapeutic approach to this disease (Fossum et al. 2012). They range from the conservative treatment of small size diverticula, to the administration of semi-liquid food served at the dog's height over a long period of time, to the surgical treatment of large diverticula. There are several techniques of surgical treatment, the main ones being esophagomyotomy, laparoscopy, esophagus substitution or the reparative procedure. Some authors recommend surgical resection of dilatations, whereas others (Fossum et al. 2012) are in favour of using biomaterials such as omentum, sternohyoid muscles, sternothyroid muscles, intercostal muscles, or diaphragmatic muscles over the parietal defect in order to increase the tightness support. There is also a possibility of using the gastric wall pediculi (Fossum et al 2012) mobilized through the diaphragm. Authors such as Paulo and Wagner (2007) like to use a diaphragmatic pediculus to repair the esophagus wall. In our case we used a knitted polyester prosthesis with small meshes, which is efficient in this type of intervention and very well tolerated both by humans and animals (Muste et al. 2011). By implanting the prosthesis in the cervical area and covering it with a part of the dilated esophagus wall, we had the possibility to restore and keep the esophageal lumen at the right diameter according to the subject's weight and height, an aspect visible two weeks after the surgery through administration of barium sulphate and radiography.

Via this procedure we were able to avoid the resection of quite a large portion of the esophagus wall and thus prevent the occurrence of scars or strictures at this particular level. The biggest advantage is that a prosthesis used in this way determines the increase 
of support and stability of the affected esophagus portion without hindering the food transit. After being replaced in its location, the operated esophagus will not develop local adhesions since the prosthesis is surrounded by its own tissue. Soon after the surgery the patient's general condition was very good. Over time, no changes could be found in the throat mobility nor any other further complications. After the surgery, the dog underwent 5 days of therapy with fluids, energizers, analgesics, and antibiotics.

During the second intervention, the prosthesis was applied differently, on the serosal face of the esophagus, like a "sleeve"; the major reason being to increase the support and safety of the esophagus walls. We used this method in order to preserve the entire esophageal mucosa as well as the entire esophageal wall because of the particularities of this organ such as its segmental vascular input, continuous movement and distension during the breathing process, plus the deglutition movements. All of these cause a high pressure over the suture lines, posing a risk of future wound dehiscence.

Inside the prosthesis the esophagus does not bear any pressure or tension whatsoever. It can perform its peristaltic movements very easily, and the inner walls of the prosthesis are perfectly smooth, enabling the passing of food. The walls of the polyester prosthesis have the necessary resistance to cope with the food transit, wall movements and distension during breathing, without the risk of breaking or dilaceration.

Another big advantage of using these prostheses is the fact that in time, the newly formed connective tissue goes very easily through the knitted prosthesis meshes, forming a uniform layer which acts as a stable structure that generates very high stability and solid support during the animal's life.

The use of these two prostheses, due to their smooth, elastic and resistant walls which take over part of the local pressure, helps prevent the tearing of the dilated walls as well as the increase of dilatations, wall resection, esophageal mucosa resection, and scar formation or dilation recurrance. It must also be emphasized that using these prostheses markedly shortens the surgery time and implicitly eases the team's efforts; the tractions over the esophagus are minimal, and the approach to the esophagus is much easier.

The use of artificial biomaterials in the reconstruction of esophageal walls has become increasingly popular, offering satisfactory results (Zhang et al. 2008). Until a successful use of artificial esophagus, the use of a polyester vascular prosthesis remains a very good option for small to medium length dilatations, as they provide protection, stability, durability without negatively influencing peristaltism (motility) or inducing any side effects.

After the surgery the subject underwent the therapeutic protocol with fluid therapy, energizers, analgesics and antibiotics for 7 days, then went on liquid alimentation for 30 days, followed by 20 more days on semi-liquid alimentation, and then gradually turned to basic alimentation. During the first 3 days post surgery we observed an inflammatory reaction of the suture area, slight but repeated coughing, shallow breathing, and avoidance of movement. All these signs gradually lessened every day, completely dissapearing 6 days after the surgery. Eleven months after the surgery, the dog's health condition was very good.

\section{References}

Fossum WT 2012: Surgery of the esophagus. In: Fossum WT (Ed.) 2012: Small Animal Surgery. $4^{\text {th }}$ ed., Mosby, pp. 372-394

Hill FW, Christie BA, Reynolds WT, Lavelle RB 1979: An oesophageal diverticulum in a dog. Aust Vet J 55: 184-187

Iwasaki M, de Alvarenga J, de Martin BW 1977: Esophageal diverticula in a dog. Mod Vet Pract 58: 606-613

Qin X, Xu ZF, Zhao XW, Shi HC, Zhou JH, Sun K, Gao XY 2003: Reconstruction of a cervical esophagus segment with an artificial prosthesis by use of a polyurethane stent covered with collagen-chitosan sponge in dogs (in Chinese). Zhonghua Wai Ke Za Zhi (Chinese Journal of Surgery) 17: 374-377

Muste A, Beteg F, Krupaci A, Muste M, Scurtu L, Bota A, Mesteşanu C, Iulian I 2011: Research and observations on the vascular prosthesis biocompatibility in pig, Scientifical Papers Veterinary Medicine 44: 6-11 
Paulo NM, Wagner M 2007: Reconstruction of thoracic esophagus with pediculated diaphragmatic flap in dogs, Acta Cir Bras 22: 17-22

Pearson H, Gibbs C, Kelly DF 1978: Oesophageal diverticulum formation in the dog. J. Small Anim Pract 19: 341-355

Shaw DH, Ihle SL 1997: Gastrointestinal disease II. Esophageal disorders. In: Wolters K (Ed.): Small Animal Internal Medicine. WB Saunders Co., Philadelphia, pp. 257-258

Zhang LJ, Rong TH, Su XD, Lin P, Long H, FU JH 2008: Experimental replacement of thoracic esophageal segment with a biomaterial artificial esophagus in dogs. J Med Coll PLA 23: 1-8 
Plate V

Muste A. N. et al.: Use of Dacron... pp. 243-247

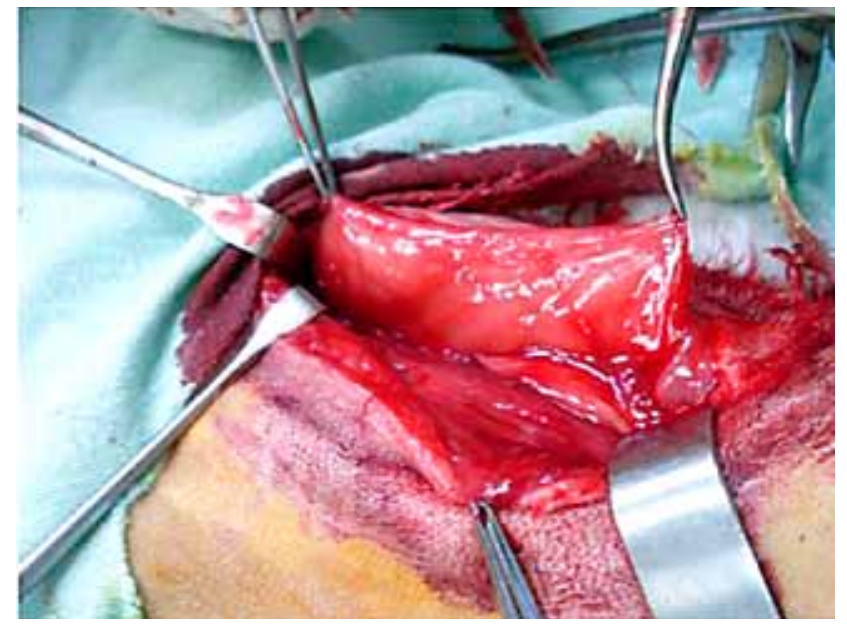

Fig. 1. Aspect of dilated cervical esophagus

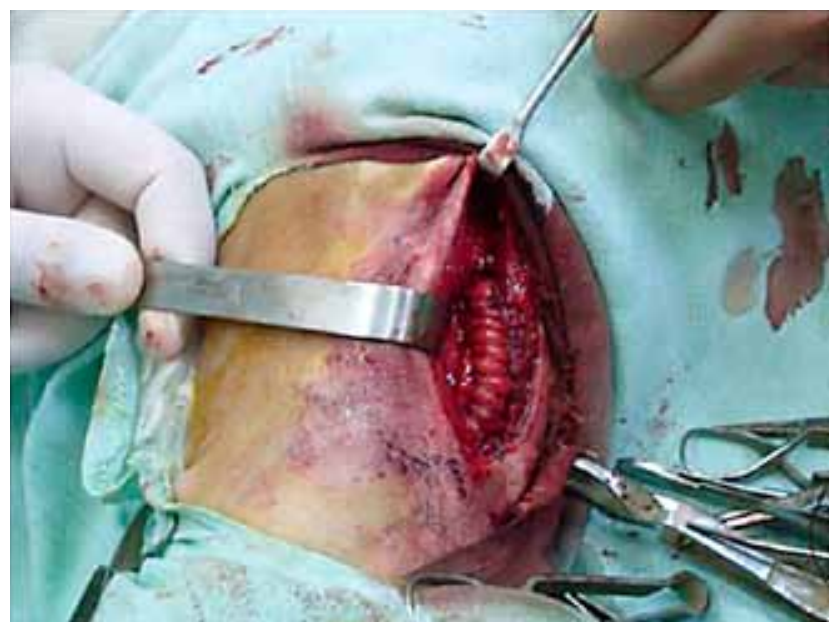

Fig. 2. Final aspect of the prosthesis suture 